\title{
Concentrations of main serum opsonins in early infancy
}

V Drossou, F Kanakoudi, E Diamanti, V Tzimouli, T Konstantinidis, A Germenis, G Kremenopoulos, V Katsougiannopoulos

\begin{abstract}
The evolution of the main serum opsonins in neonates and infants of varying gestational age was investigated to provide reference values for these opsonins in early infancy. Serum concentrations of immunoglobulins, IgG subclasses, C3, C4 and fibronectin were serially measured from birth until the age of 6 months in term and preterm infants. Measurements were performed by rate nephelometry. Five hundred and sixty six neonates (gestational age 26-41 weeks) were examined at birth, 233 at 1 month, 218 at 3 months, and 147 at 6 months, respectively. The same measurements were performed in 54 pairs of neonatal/maternal samples and in 230 apparently healthy adults. Gestational age had a significant impact on serum IgG, IgG subclasses, $\mathrm{C} 3$ and $\mathrm{C} 4$ up till the third month, and on fibronectin until the first month. No such impact was observed for IgA and IgM. Sixteen per cent of the neonates had IgM concentrations higher than $0.2 \mathrm{~g} / \mathrm{l}$ at birth, suggesting that the critical concentration of serum IgM at birth for suspected intrauterine infection should be reconsidered. Concentrations of all opsonins at birth were significantly lower than adult reference values. They only approached or even reached adult values by the third or the sixth month.

Data from analysis of the neonatal and the corresponding maternal sera indicate that there is a preferential active transplacental transport of IgG subclasses in the order of IgG1, IgG3, IgG2 and IgG4. These results show that concentrations of immunoglobulins, C3, C4 and fibronectin undergo changes during the first months of life, depending not only on the infants' postnatal age but also on gestational age. (Arch Dis Child 1995; 72: F172-F175)
\end{abstract}

Keywords: complement, fibronectin, preterm infants, immunoglobulins.

Department of Epidemiology and Hygiene

T Konstantinidis

V Katsougiannopoulos

Department of Immunology and National Tissue Typing Center, General Hospital of Athens A Germenis

Correspondence to: Vassiliki Drossou, 52 Neapoleos St, 54454 Thessaloniki, Greece.

Accepted 1 March 1995 with adult values, because of low titres of maternally derived IgG and IgG subclass specific antibodies, ${ }^{1-6}$ or because of insufficient production of $\mathrm{C} 3, \mathrm{C} 4^{7-8}$ and fibronectin. ${ }^{9-11}$ Gestational age reference values are therefore needed for the clinical interpretation of opsonin measurements in early infancy. Published values have not included all opsonins and refer to relatively small series of infants. This is probably because there are certain limitations in the determination of serum proteins in early infancy, mainly arising from the amount of blood required. However, the recent development of rapid and accurate methods requiring minimal quantities of blood, such as nephelometry, the wide application of these measurements is now practicable.

\section{Methods}

Five hundred and sixty six neonates (315 males and 251 females), appropriate for gestational age, were studied. Gestational age was determined by prenatal ultrasonography, when available, or by accurate maternal dates, and confirmed by neonatal examination (Ballard score). The infants were divided into three groups on the basis of gestational age:

Group I consisted of 99 preterm infants ranging from 26 to 30 weeks (mean gestational age 28.37 (1.4) weeks and birthweight of 1125 (264) $\mathrm{g}$.

Group II comprised 207 infants ranging from 31 to 37 weeks (mean 33.4 (1.9) weeks and birthweight of 2011 (497) $\mathrm{g}$.

Group III comprised 260 term infants (mean gestational age 39.5 (1) week and birthweight of $3440(417) \mathrm{g}$.

All neonates with evidence of intrauterine or perinatal infection, intrauterine growth retardation, major congenital abnormalities and uncertain gestational age were excluded from the study. Informed consent was obtained from all parents.

Blood samples were collected through an indwelling umbilical arterial catheter or by venepuncture during routine procedures within the first 24 hours of birth. Blood samples were also collected from 54 mothers 12 hours after delivery and their corresponding neonates of 37-42 weeks' gestation.

Of the 566 infants enrolled in the study, 233, 218 , and 147 were followed up to the end of the first, third, and sixth month, respectively. Infants who had either received intravenous immunoglobulin or double volume exchange transfusion, or who developed infections during the study period were excluded from the follow up. Follow up included clinical examination, collection of data about infections, and measurement of serum opsonin concentrations. 
Table 1 Percentiles of serum concentrations of immunoglobulins in three groups of infants from birth until 6 months of age

\begin{tabular}{|c|c|c|c|c|c|c|c|c|c|c|c|c|c|c|c|c|c|}
\hline \multirow{2}{*}{$\begin{array}{l}\text { Postnatal } \\
\text { age }\end{array}$} & \multirow[b]{2}{*}{ Group } & \multirow[b]{2}{*}{$n=$} & \multicolumn{5}{|c|}{ IgG percentiles } & \multicolumn{5}{|c|}{ IgM percentiles } & \multicolumn{5}{|c|}{ IgA percentiles } \\
\hline & & & 5 & 25 & 50 & 75 & 95 & 5 & 25 & 50 & 75 & 95 & 5 & 25 & 50 & 75 & 95 \\
\hline rth & $\begin{array}{l}\text { I } \\
\text { II } \\
\text { III } \\
p^{\star}\end{array}$ & $\begin{array}{r}99 \\
207 \\
260\end{array}$ & $\begin{array}{l}2 \cdot 80 \\
5.08 \\
6.90\end{array}$ & $\begin{array}{l}3 \cdot 88 \\
6 \cdot 64 \\
9 \cdot 65\end{array}$ & $\begin{array}{c}4.96 \\
8.02 \\
11.40 \\
<0.001\end{array}$ & $\begin{array}{r}5.93 \\
9.87 \\
12.85\end{array}$ & $\begin{array}{r}7 \cdot 53 \\
12 \cdot 19 \\
15 \cdot 32\end{array}$ & $\begin{array}{l}0 \cdot 0 \\
0 \cdot 0 \\
0 \cdot 07\end{array}$ & $\begin{array}{l}0.06 \\
0.04 \\
0.09\end{array}$ & $\begin{array}{c}0.08 \\
0.09 \\
0.13 \\
<0.001\end{array}$ & $\begin{array}{l}0 \cdot 16 \\
0 \cdot 15 \\
0 \cdot 17\end{array}$ & $\begin{array}{l}0.36 \\
0.31 \\
0.28\end{array}$ & & & & & \\
\hline 1 month & $\begin{array}{l}\text { I } \\
\text { II } \\
\text { III } \\
p^{\star}\end{array}$ & $\begin{array}{r}54 \\
104 \\
75\end{array}$ & $\begin{array}{l}2 \cdot 25 \\
2 \cdot 84 \\
4 \cdot 16\end{array}$ & $\begin{array}{l}3 \cdot 21 \\
3 \cdot 71 \\
5 \cdot 35\end{array}$ & $\begin{array}{l}3.75 \\
4.50 \\
6.23 \\
<0.001\end{array}$ & $\begin{array}{l}4 \cdot 22 \\
5 \cdot 36 \\
7 \cdot 44\end{array}$ & $\begin{array}{r}5 \cdot 60 \\
7 \cdot 56 \\
10 \cdot 17\end{array}$ & $\begin{array}{l}0.25 \\
0 \cdot 22 \\
0 \cdot 21\end{array}$ & $\begin{array}{l}0.41 \\
0.39 \\
0.39 \\
\text { NS }\end{array}$ & $\begin{array}{l}0.48 \\
0.47 \\
0.59\end{array}$ & $\begin{array}{l}0.68 \\
0.65 \\
0.79\end{array}$ & $\begin{array}{l}0.89 \\
0.88 \\
1.05\end{array}$ & $\begin{array}{l}0.0 \\
0.0 \\
0.0\end{array}$ & $\begin{array}{l}0.08 \\
0.0 \\
0 \cdot 10\end{array}$ & $\begin{array}{r}0.12 \\
0.09 \\
0.12 \\
<0.05\end{array}$ & $\begin{array}{l}0.17 \\
0.13 \\
0.21\end{array}$ & $\begin{array}{l}0.26 \\
0.29 \\
0.32\end{array}$ \\
\hline 3 months & $\begin{array}{l}\text { I } \\
\text { II } \\
\text { III } \\
p^{\star}\end{array}$ & $\begin{array}{r}54 \\
104 \\
60\end{array}$ & $\begin{array}{l}1 \cdot 07 \\
1 \cdot 20 \\
2 \cdot 75\end{array}$ & $\begin{array}{l}1 \cdot 94 \\
2 \cdot 11 \\
3 \cdot 76\end{array}$ & $\begin{array}{c}2.50 \\
3.09 \\
4.06 \\
<0.001\end{array}$ & $\begin{array}{l}3 \cdot 27 \\
3 \cdot 83 \\
4 \cdot 82\end{array}$ & $\begin{array}{l}4 \cdot 48 \\
5 \cdot 80 \\
7 \cdot 06\end{array}$ & $\begin{array}{l}0.28 \\
0.35 \\
0.43\end{array}$ & $\begin{array}{l}0.45 \\
0.52 \\
0.68\end{array}$ & $\begin{array}{r}0.58 \\
0.69 \\
0.86 \\
<0.01\end{array}$ & $\begin{array}{l}0.77 \\
0.97 \\
1.09\end{array}$ & $\begin{array}{l}1.30 \\
1.41 \\
1.46\end{array}$ & $\begin{array}{l}0 \cdot 0 \\
0 \cdot 01 \\
0 \cdot 11\end{array}$ & $\begin{array}{l}0.13 \\
0.09 \\
0.18\end{array}$ & $\begin{array}{l}0 \cdot 19 \\
0 \cdot 19 \\
0 \cdot 24 \\
\text { NS }\end{array}$ & $\begin{array}{l}0.23 \\
0 \cdot 24 \\
0 \cdot 32\end{array}$ & $\begin{array}{l}0.35 \\
0.41 \\
0.39\end{array}$ \\
\hline 6 months & $\begin{array}{l}\text { I } \\
\text { II } \\
\text { III } \\
p^{\star}\end{array}$ & $\begin{array}{l}35 \\
79 \\
33\end{array}$ & $\begin{array}{l}2 \cdot 03 \\
2 \cdot 05 \\
3 \cdot 47\end{array}$ & $\begin{array}{l}3 \cdot 40 \\
2 \cdot 97 \\
4 \cdot 30\end{array}$ & $\begin{array}{l}3.97 \\
4 \cdot 17 \\
4 \cdot 91 \\
\text { NS }\end{array}$ & $\begin{array}{l}4.65 \\
5.08 \\
6.09\end{array}$ & $\begin{array}{l}6 \cdot 71 \\
7 \cdot 51 \\
7 \cdot 30\end{array}$ & $\begin{array}{l}0.57 \\
0.42 \\
0.60\end{array}$ & $\begin{array}{l}0.79 \\
0.67 \\
0.80\end{array}$ & $\begin{array}{c}0.96 \\
0.99 \\
0.95 \\
\text { NS }\end{array}$ & $\begin{array}{l}1 \cdot 26 \\
1 \cdot 30 \\
1 \cdot 15\end{array}$ & $\begin{array}{l}1.77 \\
1.72 \\
1.62\end{array}$ & $\begin{array}{l}0.06 \\
0.01 \\
0 \cdot 16\end{array}$ & $\begin{array}{l}0.17 \\
0.15 \\
0.22\end{array}$ & $\begin{array}{l}0 \cdot 27 \\
0 \cdot 22 \\
0 \cdot 26 \\
\text { NS }\end{array}$ & $\begin{array}{l}0.35 \\
0.39 \\
0.35\end{array}$ & $\begin{array}{l}0.44 \\
0.62 \\
0.43\end{array}$ \\
\hline Adults & & 230 & $8 \cdot 74$ & $10 \cdot 80$ & $12 \cdot 30$ & $14 \cdot 20$ & 16.90 & 0.64 & 1.03 & $1 \cdot 40$ & $1 \cdot 86$ & 2.99 & 1.03 & $1 \cdot 60$ & 2.05 & $2 \cdot 54$ & $3 \cdot 47$ \\
\hline
\end{tabular}

*Significance of difference among the three groups using the Kruskal-Wallis one way analysis of variance; NS not significant.

Serum and plasma samples were stored at $-70^{\circ} \mathrm{C}$ until analysed. All parameters were measured in serum except for fironectin which was measured in plasma. All measurements were performed by rate nephelometry - fixed time nephelometry - using the Behring Nephelometer Analyser (BNA). Data obtained from 230 healthy blood donors were used as adult reference values.

\section{STATISTICAL ANALYSIS}

Percentiles (5th, 25th, 50th, 75th, and 95th) of the measured variables for each gestational age group at birth and at each postnatal age were calculated. The Kruskal-Wallis one way analysis of variance was used to evaluate the difference of values among various gestational age groups. The Wilcoxon rank sum test for paired samples was used to compare the respective concentrations in neonatal and maternal sera. ${ }^{12}$ Data were analysed using the SPSS for MS Windows (version 5.0.1, International Use, SPSS Company) software package.

\section{Results}

Total IgG subclass values at birth differed significantly among the different groups (tables
1 and 2). A wide range of individual values of IgG2 and IgG4 was observed. The relative concentrations of IgG subclasses were similar in all gestational age groups $(70 \%, 20 \%, 7 \%$ and $3 \%$ for IgG1, IgG2, IgG3 and IgG4, respectively). Serial IgG measurements showed that concentrations decreased progressively until the third month in all gestational age groups, and then increased again until the sixth month (table 1). The pattern of IgG1 was similar to that of IgG, while IgG3 reached the lowest concentrations at the end of the first month and increased thereafter. IgG2 and IgG4 concentrations declined during the first three months and remained low until the sixth month (table 2).

IgA was undetectable by rate nephelometry $(<0.07 \mathrm{~g} / \mathrm{l})$ in $99 \%$ of the infants at birth, but at six months of age it could be measured in all infants.

IgM concentrations at birth showed a wide range of individual values. In 90 of the 566 $(16 \%)$ neonates, values were higher than $0 \cdot 2$ $\mathrm{g} / \mathrm{l}$. Concentrations increased rapidly, reaching adult reference values by six months (table 1).

At birth, mean concentrations of $\mathrm{C} 3$ and $\mathrm{C} 4$ corresponded to $0.60-0.79$ and $0.41-0.59$ of the respective adult reference values, depending on gestational age. During the following months, concentrations of these complement

Table 2 Percentiles of serum concentrations of IgG subclasses in three groups of infants from birth until 6 months of age

\begin{tabular}{|c|c|c|c|c|c|c|c|c|c|c|c|c|c|c|c|c|c|c|c|c|c|c|}
\hline \multirow{2}{*}{$\begin{array}{l}\text { Postnatal } \\
\text { age }\end{array}$} & \multirow[b]{2}{*}{ Group } & \multirow[b]{2}{*}{$n=$} & \multicolumn{5}{|c|}{ IgG1 percentiles } & \multicolumn{5}{|c|}{ IgG2 percentiles } & \multicolumn{5}{|c|}{ IgG3 percentiles } & \multicolumn{5}{|c|}{ IgG4 percentiles } \\
\hline & & & 5 & 25 & 50 & 75 & 95 & 5 & 25 & 50 & 75 & 95 & 5 & 25 & 50 & 75 & 95 & 5 & 25 & 50 & 75 & 95 \\
\hline Birth & $\begin{array}{l}\text { I } \\
\text { II } \\
\text { III } \\
p^{\star}\end{array}$ & $\begin{array}{r}99 \\
207 \\
260\end{array}$ & $\begin{array}{l}1.48 \\
0.76 \\
3.36\end{array}$ & $\begin{array}{l}2 \cdot 08 \\
3 \cdot 88 \\
5 \cdot 81\end{array}$ & $\begin{array}{c}3.11 \\
5.13 \\
7.41 \\
<0.001\end{array}$ & $\begin{array}{l}3.99 \\
6.59 \\
8.93\end{array}$ & $\begin{array}{l}6 \cdot 16 \\
8 \cdot 59 \\
12 \cdot 0\end{array}$ & $\begin{array}{l}0.0 \\
0.40 \\
0.67\end{array}$ & $\begin{array}{l}0.60 \\
0.86 \\
1.36\end{array}$ & $\begin{array}{c}0.85 \\
1.32 \\
2 \cdot 14 \\
<0.001\end{array}$ & $\begin{array}{l}1 \cdot 16 \\
1 \cdot 80 \\
3 \cdot 02\end{array}$ & $\begin{array}{l}1 \cdot 60 \\
3 \cdot 01 \\
5 \cdot 22\end{array}$ & $\begin{array}{l}0 \cdot 13 \\
0 \cdot 16 \\
0 \cdot 26\end{array}$ & $\begin{array}{l}0 \cdot 19 \\
0 \cdot 37 \\
0 \cdot 48\end{array}$ & $\begin{array}{c}0.26 \\
0.49 \\
0.70 \\
<0.001\end{array}$ & $\begin{array}{l}0.41 \\
0.66 \\
0.93\end{array}$ & $\begin{array}{l}0.55 \\
1.01 \\
1.48\end{array}$ & $\begin{array}{l}0.0 \\
0.0 \\
0.0\end{array}$ & $\begin{array}{l}0.0 \\
0.08 \\
0.09\end{array}$ & $\begin{array}{c}0.11 \\
0.18 \\
0.23 \\
<0.001\end{array}$ & $\begin{array}{l}0 \cdot 19 \\
0 \cdot 34 \\
0 \cdot 44\end{array}$ & $\begin{array}{l}0.35 \\
0.67 \\
0.97\end{array}$ \\
\hline 1 month & $\begin{array}{l}\text { I } \\
\text { II } \\
\text { III } \\
p^{\star}\end{array}$ & $\begin{array}{r}54 \\
104 \\
75\end{array}$ & $\begin{array}{l}1 \cdot 17 \\
1 \cdot 20 \\
2 \cdot 76\end{array}$ & $\begin{array}{l}1 \cdot 85 \\
2 \cdot 20 \\
3 \cdot 36\end{array}$ & $\begin{array}{c}2.20 \\
2.92 \\
3.90 \\
<0.001\end{array}$ & $\begin{array}{l}2.86 \\
3.79 \\
4.89\end{array}$ & $\begin{array}{l}4 \cdot 54 \\
5 \cdot 08 \\
6 \cdot 36\end{array}$ & $\begin{array}{l}0.34 \\
0.10 \\
0.43\end{array}$ & $\begin{array}{l}0.67 \\
0.96 \\
0.76\end{array}$ & $\begin{array}{c}0.86 \\
1.16 \\
1.11 \\
<0.001\end{array}$ & $\begin{array}{l}1.07 \\
0.63 \\
1.47\end{array}$ & $\begin{array}{l}1.64 \\
1.69 \\
2.54\end{array}$ & $\begin{array}{l}0.04 \\
0 \cdot 16 \\
0 \cdot 20\end{array}$ & $\begin{array}{l}0 \cdot 16 \\
0 \cdot 22 \\
0 \cdot 26\end{array}$ & $\begin{array}{r}0.24 \\
0.28 \\
0.32 \\
<0.01\end{array}$ & $\begin{array}{l}0.30 \\
0.39 \\
0.46\end{array}$ & $\begin{array}{l}0.42 \\
0.62 \\
0.71\end{array}$ & $\begin{array}{l}0.0 \\
0.0 \\
0.0\end{array}$ & $\begin{array}{l}0.0 \\
0.0 \\
0.0\end{array}$ & $\begin{array}{r}0.11 \\
0.08 \\
0.09 \\
<0.05\end{array}$ & $\begin{array}{l}0 \cdot 16 \\
0 \cdot 17 \\
0 \cdot 20\end{array}$ & $\begin{array}{l}0.26 \\
0.31 \\
0.34\end{array}$ \\
\hline 3 months & $\begin{array}{l}\text { I } \\
\text { II } \\
\text { III } \\
p^{\star}\end{array}$ & $\begin{array}{r}54 \\
104 \\
60\end{array}$ & $\begin{array}{l}0.60 \\
0.60 \\
1.51\end{array}$ & $\begin{array}{l}1.02 \\
1.35 \\
2 \cdot 02\end{array}$ & $\begin{array}{c}1.43 \\
1.89 \\
2.57 \\
<0.001\end{array}$ & $\begin{array}{l}1.89 \\
2.76 \\
3.36\end{array}$ & $\begin{array}{l}3.95 \\
3.82 \\
5 \cdot 13\end{array}$ & $\begin{array}{l}0 \cdot 0 \\
0 \cdot 0 \\
0 \cdot 0\end{array}$ & $\begin{array}{l}0.0 \\
0.0 \\
0.48\end{array}$ & $\begin{array}{c}0.45 \\
0.55 \\
0.67 \\
<0.001\end{array}$ & $\begin{array}{l}0 \cdot 75 \\
0 \cdot 76 \\
0 \cdot 79\end{array}$ & $\begin{array}{l}1.04 \\
1.17 \\
1.36\end{array}$ & $\begin{array}{l}0 \cdot 0 \\
0 \cdot 11 \\
0 \cdot 13\end{array}$ & $\begin{array}{l}0 \cdot 11 \\
0 \cdot 16 \\
0 \cdot 26\end{array}$ & $\begin{array}{c}0.18 \\
0.27 \\
0.32 \\
<0.001\end{array}$ & $\begin{array}{l}0.34 \\
0.46 \\
0.46\end{array}$ & $\begin{array}{l}0.57 \\
0.82 \\
0.92\end{array}$ & $\begin{array}{l}0.0 \\
0 \cdot 0 \\
0.0\end{array}$ & $\begin{array}{l}0 \cdot 0 \\
0 \cdot 0 \\
0 \cdot 0\end{array}$ & $\begin{array}{l}0 \cdot 0 \\
0 \cdot 0 \\
0.0 \\
\text { NS }\end{array}$ & $\begin{array}{l}0 \cdot 0 \\
0 \cdot 0 \\
0 \cdot 0\end{array}$ & $\begin{array}{l}0.09 \\
0.04 \\
0.10\end{array}$ \\
\hline 6 months & $\begin{array}{l}\text { I } \\
\text { II } \\
\text { III } \\
p^{\star}\end{array}$ & $\begin{array}{l}35 \\
79 \\
33\end{array}$ & $\begin{array}{l}0 \cdot 78 \\
1 \cdot 17 \\
2 \cdot 10\end{array}$ & $\begin{array}{l}2 \cdot 02 \\
1 \cdot 81 \\
2 \cdot 43\end{array}$ & $\begin{array}{l}2 \cdot 57 \\
2 \cdot 86 \\
3.68 \\
\text { NS }\end{array}$ & $\begin{array}{l}3 \cdot 24 \\
3 \cdot 79 \\
4 \cdot 38\end{array}$ & $\begin{array}{l}4 \cdot 94 \\
5 \cdot 86 \\
5 \cdot 84\end{array}$ & $\begin{array}{l}0 \cdot 0 \\
0 \cdot 0 \\
0 \cdot 0\end{array}$ & $\begin{array}{l}0.0 \\
0.0 \\
0.0\end{array}$ & $\begin{array}{l}0.45 \\
0.40 \\
0.40 \\
\text { NS }\end{array}$ & $\begin{array}{l}0.75 \\
0.76 \\
0.67\end{array}$ & $\begin{array}{l}1.09 \\
0.95 \\
1.62\end{array}$ & $\begin{array}{l}0.01 \\
0.04 \\
0.18\end{array}$ & $\begin{array}{l}0.22 \\
0.26 \\
0.32\end{array}$ & $\begin{array}{l}0.33 \\
0.39 \\
0.45 \\
\text { NS }\end{array}$ & $\begin{array}{l}0.49 \\
0.54 \\
0.75\end{array}$ & $\begin{array}{l}0.78 \\
0.86 \\
1.00\end{array}$ & $\begin{array}{l}0.0 \\
0 \cdot 0 \\
0.0\end{array}$ & $\begin{array}{l}0.0 \\
0.0 \\
0.0\end{array}$ & $\begin{array}{l}0.0 \\
0.0 \\
0.0 \\
\text { NS }\end{array}$ & $\begin{array}{l}0 \cdot 0 \\
0 \cdot 0 \\
0 \cdot 0\end{array}$ & $\begin{array}{l}0 \cdot 07 \\
0 \cdot 10 \\
0 \cdot 12\end{array}$ \\
\hline Adults & & 50 & $2 \cdot 98$ & $4 \cdot 60$ & $5 \cdot 63$ & $7 \cdot 49$ & $9 \cdot 86$ & $1 \cdot 32$ & $2 \cdot 20$ & $2 \cdot 73$ & $3 \cdot 81$ & $5 \cdot 61$ & $0 \cdot 31$ & 0.54 & 0.68 & 0.83 & $1 \cdot 25$ & 0.0 & 0.25 & 0.40 & 0.75 & 1.02 \\
\hline
\end{tabular}

^Significance of difference among the three groups using the Kruskal-Wallis one way analysis of variance; NS not significant. 
Table 3 Percentiles of serum concentrations of C3, C4, and fibronectin in three groups of infants from birth until 6 months of age

\begin{tabular}{|c|c|c|c|c|c|c|c|c|c|c|c|c|c|c|c|c|c|}
\hline \multirow{2}{*}{$\begin{array}{l}\text { Postnatal } \\
\text { age }\end{array}$} & \multirow[b]{2}{*}{ Group } & \multirow[b]{2}{*}{$n=$} & \multicolumn{5}{|c|}{ C3 percentiles } & \multicolumn{5}{|c|}{ C4 percentiles } & \multicolumn{5}{|c|}{ Fibronectin percentiles } \\
\hline & & & 5 & 25 & 50 & 75 & 95 & 5 & 25 & 50 & 75 & 95 & 5 & 25 & 50 & 75 & 95 \\
\hline Birth & $\begin{array}{l}\text { I } \\
\text { II } \\
\text { III } \\
p^{\star}\end{array}$ & $\begin{array}{r}68 \\
131 \\
260\end{array}$ & & $\begin{array}{l}0.37 \\
0.45\end{array}$ & $\begin{array}{c}0.37 \\
0.47 \\
0.53 \\
<0.001\end{array}$ & $\begin{array}{l}0.6 \\
0 \cdot 5 \\
0.6\end{array}$ & & $\begin{array}{l}0 \cdot 04 \\
0 \cdot 08 \\
0 \cdot 10\end{array}$ & $\begin{array}{l}0.09 \\
0.12 \\
0.13\end{array}$ & $\begin{array}{c}0.11 \\
0.13 \\
0.15 \\
<0.001\end{array}$ & $\begin{array}{l}0 \cdot 15 \\
0 \cdot 17 \\
0 \cdot 18\end{array}$ & $\begin{array}{l}0 \cdot 24 \\
0 \cdot 24 \\
0 \cdot 24\end{array}$ & $\begin{array}{l}0.08 \\
0.08 \\
0.09\end{array}$ & $\begin{array}{l}0.09 \\
0 \cdot 10 \\
0.12\end{array}$ & $\begin{array}{r}0.11 \\
0.13 \\
0.15 \\
<0.05\end{array}$ & $\begin{array}{l}0.14 \\
0.17 \\
0 \cdot 18\end{array}$ & $\begin{array}{l}0.19 \\
0.21 \\
0.23\end{array}$ \\
\hline 1 month & $\begin{array}{l}\text { I } \\
\text { II } \\
\text { III } \\
p^{\star}\end{array}$ & $\begin{array}{l}37 \\
68 \\
76\end{array}$ & $\begin{array}{l}0.30 \\
0.32 \\
0.41\end{array}$ & $\begin{array}{l}0 \cdot 37 \\
0 \cdot 44 \\
0 \cdot 54\end{array}$ & $\begin{array}{c}0.53 \\
0.50 \\
0.64 \\
<0.001\end{array}$ & $\begin{array}{l}0.69 \\
0.62 \\
0.72\end{array}$ & $\begin{array}{l}0.85 \\
0 \cdot 81 \\
0 \cdot 90\end{array}$ & $\begin{array}{l}0 \cdot 10 \\
0 \cdot 11 \\
0 \cdot 12\end{array}$ & $\begin{array}{l}0.12 \\
0.13 \\
0 \cdot 19\end{array}$ & $\begin{array}{c}0.17 \\
0.16 \\
0.22 \\
<0.001\end{array}$ & $\begin{array}{l}0 \cdot 23 \\
0 \cdot 22 \\
0 \cdot 27\end{array}$ & $\begin{array}{l}0 \cdot 32 \\
0 \cdot 34 \\
0 \cdot 36\end{array}$ & $\begin{array}{l}0.11 \\
0.11 \\
0.13\end{array}$ & $\begin{array}{l}0.16 \\
0.18 \\
0.18\end{array}$ & $\begin{array}{l}0 \cdot 21 \\
0 \cdot 23 \\
0 \cdot 22 \\
\text { NS }\end{array}$ & $\begin{array}{l}0 \cdot 24 \\
0 \cdot 28 \\
0 \cdot 29\end{array}$ & $\begin{array}{l}0.34 \\
0.37 \\
0.41\end{array}$ \\
\hline $3 \mathrm{~m}$ & $\begin{array}{l}\text { I } \\
\text { II } \\
\text { III } \\
p^{\star}\end{array}$ & $\begin{array}{l}35 \\
61 \\
60\end{array}$ & $\begin{array}{l}0.35 \\
0.48 \\
0.55\end{array}$ & $\begin{array}{l}0.49 \\
0.59 \\
0.69\end{array}$ & $\begin{array}{l}0.64 \\
0.70 \\
0.76 \\
<0.001\end{array}$ & $\begin{array}{l}0.73 \\
0.77 \\
0.87\end{array}$ & $\begin{array}{l}0.95 \\
0.92 \\
1.02\end{array}$ & $\begin{array}{l}0 \cdot 11 \\
0 \cdot 11 \\
0 \cdot 16\end{array}$ & $\begin{array}{l}0.13 \\
0.16 \\
0.22\end{array}$ & $\begin{array}{r}0.16 \\
0.22 \\
0.26 \\
<0.01\end{array}$ & $\begin{array}{l}0 \cdot 25 \\
0 \cdot 28 \\
0 \cdot 32\end{array}$ & $\begin{array}{l}0.31 \\
0 \cdot 37 \\
0 \cdot 47\end{array}$ & $\begin{array}{l}0 \cdot 14 \\
0 \cdot 17 \\
0 \cdot 18\end{array}$ & $\begin{array}{l}0 \cdot 24 \\
0 \cdot 22\end{array}$ & $\begin{array}{l}0.25 \\
0 \cdot 28 \\
0 \cdot 30 \\
\text { NS }\end{array}$ & $\begin{array}{l}0.33 \\
0.33 \\
0.35\end{array}$ & $\begin{array}{l}0.36 \\
0.39 \\
0.45\end{array}$ \\
\hline 6 months & $\begin{array}{l}\text { I } \\
\text { II } \\
\text { III } \\
p^{\star}\end{array}$ & $\begin{array}{l}19 \\
38 \\
33\end{array}$ & $\begin{array}{l}0.75 \\
0.62 \\
0.64\end{array}$ & $\begin{array}{l}0 \cdot 80 \\
0 \cdot 71 \\
0 \cdot 76\end{array}$ & $\begin{array}{l}0 \cdot 85 \\
0 \cdot 82 \\
0 \cdot 87 \\
\text { NS }\end{array}$ & $\begin{array}{l}0.97 \\
0.94 \\
1.01\end{array}$ & $\begin{array}{l}1 \cdot 12 \\
1.05 \\
1.24\end{array}$ & $\begin{array}{l}0 \cdot 18 \\
0 \cdot 16 \\
0 \cdot 18\end{array}$ & $\begin{array}{l}0 \cdot 21 \\
0.23 \\
0.23\end{array}$ & $\begin{array}{l}0.23 \\
0 \cdot 31 \\
0 \cdot 31 \\
\text { NS }\end{array}$ & $\begin{array}{l}0 \cdot 27 \\
0 \cdot 39 \\
0 \cdot 40\end{array}$ & $\begin{array}{l}0.32 \\
0.47 \\
0.55\end{array}$ & $\begin{array}{l}0 \cdot 20 \\
0 \cdot 24 \\
0 \cdot 23\end{array}$ & $\begin{array}{l}0 \cdot 21 \\
0 \cdot 29 \\
0 \cdot 28\end{array}$ & $\begin{array}{l}0 \cdot 32 \\
0 \cdot 31 \\
0 \cdot 31 \\
\text { NS }\end{array}$ & $\begin{array}{l}0.35 \\
0.35 \\
0 \cdot 37\end{array}$ & $\begin{array}{l}0.40 \\
0.40 \\
0.44\end{array}$ \\
\hline Adults & & 230 & 0.49 & 0.64 & 0.72 & $0 \cdot 81$ & 1.03 & $0 \cdot 15$ & 0.22 & $0 \cdot 26$ & 0.32 & 0.41 & 0.08 & $0 \cdot 26$ & 0.34 & 0.43 & 0.63 \\
\hline
\end{tabular}

^Significance of difference among the three groups using the Kruskal-Wallis one way analysis of variance; NS not significant.

components increased, reaching adult values by the third or sixth month of age. A wide variation of values within each gestational age group was evident especially among neonates with a gestational age of less than 35 weeks (table 3).

Fibronectin concentrations were less than half of the adult values at birth and then showed a rapid increase, approaching adult reference values by the sixth month (table 3 ).

The significance of the difference in opsonin concentrations among the various gestational age groups is shown in tables 1 to 3 .

Statistical analysis of the results obtained from 54 paired neonatal and maternal sera showed that IgG2 and IgG3 titres in neonates were similar to those of their mothers. Significant differences were observed for total IgG, IgG1, IgG4, IgM, C3, C4 and fibronectin $(\mathrm{p}<0.01)$. The neonatal: maternal concentration ratio for IgG, IgG1, IgG2, IgG3, IgG4, $\mathrm{C} 3, \mathrm{C} 4$ and fibronectin was $1.22,1.41,0.97$, $1.08,0.86,0.59,0.49$, and 0.52 , respectively.

\section{Discussion}

Gestational age is expected to influence mainly the serum concentrations of those opsonins which are actively transferred across the placenta - namely, IgG and IgG subclasses. The impact of gestational age on the other non specific opsonins, such as C3, C4, and fibronectin, has not been adequately investigated. ${ }^{13-16}$ In our study serial measurements of these opsonins showed that gestational age had a significant impact on concentrations of IgG, IgG subclasses, $\mathrm{C} 3$ and $\mathrm{C} 4$ until the third month. This impact was not extended further than the first month on fibronectin. On the other hand, gestational age had no significant impact on IgA and IgM concentrations either at birth or subsequently.

Findings regarding the transplacental transfer of IgG subclasses are controversial. ${ }^{2} 351718$ Hay et al ${ }^{18}$ reported an impairment in transplacental transfer of IgG2 while more recent studies generally indicate that all IgG subclasses are freely transferred through the placenta. ${ }^{23517}$ Our data indicate that there is a preferential active transplacental transport of IgG subclasses in the order of IgG1, IgG3, IgG2, and IgG4. Furthermore, results from serial measurements of IgG subclasses suggest that IgG3 is produced earlier than IgG2 and IgG4. This supports the findings of Morell et al ${ }^{17}$ and Oxelious et al ,9 who studied healthy infants ranging in age from 10 days to 4 months.

It is worth noting that IgM concentrations at birth were higher than $0.2 \mathrm{~g} / 1$ in $16 \%$ of our neonates, a level so far considered to be the normal upper limit of normal for this age. ${ }^{20}$ These neonates were neither small for gestational age nor had any evidence of intrauterine infection. Similar results were obtained by Conway et $a l^{21}$ in about $35 \%$ of the studied neonates. These findings suggest that the critical level of serum IgM at birth for suspected intrauterine infection should be reconsidered.

So far published data do not provide reference values for $\mathrm{C} 3$ and $\mathrm{C} 4$ in early infancy. We found that $\mathrm{C} 3$ and $\mathrm{C} 4$ concentrations at birth were around half to two thirds those of adult reference values, depending on gestational age. Values increased with postnatal age, reaching adult values by the third or sixth month and not by the second year or later in childhood, as has been reported before. ${ }^{131422}$ As in earlier relevant studies ${ }^{1315}$ we also observed a wide range of individual values even within each gestational age group.

Data concerning fibronectin in early infancy are also limited. Two earlier papers report that fibronectin concentrations were determined in only small groups of infants ranging from 1 to 8 months of age. ${ }^{1016}$ In our study fibronectin concentrations at birth were around half the adult values, but serial measurements showed a rapidly rising pattern.

In general, reported values of IgG, IgG subclasses, $\mathrm{C} 3, \mathrm{C} 4$ and fibronectin vary widely. ${ }^{34910131423}$ A major factor for this could be the different individual methods of measurement used. We used rate nephelometry, which is a reliable and accurate method and produces results within a few hours. Data on measurements of serum opsonins using this method are very limited. ${ }^{10}$ Valetta et al, ${ }^{10}$ who also used nephelometry to measure fibronectin 
in the cord blood of 76 term and preterm neonates, reported values comparable with those of our study. From both studies it is evident that fibronectin values determined by nephelometry are higher than those found by other methods, such as electroimmunoassay $^{911}$ and enzyme immunoassay. ${ }^{16}$ On the contrary, concentrations of $\mathrm{C} 3$ and $\mathrm{C} 4$ measured by nephelometry in our study were lower than those previously reported. ${ }^{13} 14 \mathrm{We}$ believe that rate nephelometry is an appropriate method for assessing complement components because it is not affected by the presence of breakdown products.

In conclusion, our results show that concentrations of immunoglobulins, C3, C4 and fibronectin undergo significant changes during the first months of life, depending not only on the infants' postnatal age but also on their gestational age. Therefore, gestational age reference values are required for clinical interpretation of the values of these opsonins in early infancy. We believe that our results circumscribe the evolving pattern of the main serum opsonins, especially that of the IgG subclasses, C3, C4 and fibronectin.

1 Hill HR. Host defences in the neonate: prospects for enhancement. Semin Perinatol 1985; 9: 2-11.

2 Einhorn MS, Granoff DM, Nahm MH, Quinn A Shackelford PG. Concentrations of antibodies in paired maternal and infant sera: Relationship of IgG subclass. f Pediatr 1987; 111: 783-8.

3 Gasparoni A, Avanzini A, Ravagni Probizer F, Chirico G Rondini G, Severi F. IgG subclasses compared in maternal and cord serum and breast milk. Arch Dis Child 1992; 67: 41-3.

4 Evans HE, Alpata SO, Glass L. Serum immunoglobulin levels in premature and full-term infants. Am $\mathcal{F}$ Clin Pathol 1971; 56: 416-8.

5 Pitcher-Wilmott RW, Hindocha P, Wood CB. The placental transfer of IgG subclasses in human pregnancy. Clin Exp Immunol 1980; 41: 303-8.
6 Oxelious VA, Svenningsen NW. IgG subclass concentrations in preterm neonates. Acta Paediatrica Scandinavica 1984; 73: 626-30.

7 Colten HR, Goldberger G. Ontogeny of serum complement proteins. Pediatrics 1979; 64: S775-80.

8 Sawyer MK, Forman ML, Kuplic LS, Stiehm ER Developmental aspects of the human complement system. Biol Neonate 1971; 19: 148-62.

9 Domula M, Bykowska K, Wegrzynowicz Z, Lopaciuk S,

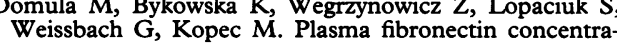
tions in healthy and septic infants. Eur $\mathcal{F}$ Paediatr 1985; 144: 49-52.

10 Valletta EA, Bonazzi L, Zuanazzi R, Del Col G, Andreoli A, Stocchero L, et al. Plasma fibronectin concentrations in healthy newborns and children. Eur $\mathcal{F}$ Paediatr 1988; 197 68-70.

11 Yoder MC, Douglas SD, Gerdes J, Kline J, Polin RA Plasma fibronectin in healthy newborn infants: Respiratory distress syndrome and perinatal asphyxia. f Pediatr 1983; 102: 777-80

12 Armitage P, Berry G. Statistical methods in medical research. Oxford: Blackwell Scientific Publications, 1987; 408-20.

13 Norman ME, Gall EP, Taylor A, Laster L, Nilsson UR. Serum complement profiles in infants and children. Serum complement profict $1975 ; 87: 912-6$.

14 Fireman P, Zuchowsky DA, Taylor PM. Development of human complement system. F Immunol 1969; 103: 25-31.

15 Roach B, Kim Y, Jerome E, Michael AF. Influence of age and sex on serum complement components in children. Am $\mathcal{F}$ Dis Child 1981; 135: 918-20.

16 McCafferty MH, Lepow M, Saba TM, Cho E, Meuwissen $\mathrm{H}$, White $\mathrm{S}$, et al. Normal fibronectin levels as a function of age in the pediatric population. Pediatr Res 1983; 17: $482-5$.

17 Morell A, Skvaril F, Hitzig WH, Barandum S. IgG subclasses: development of the serum concentrations in 'normal' infants and children. $\mathcal{f}$ Pediatr 1972; 80: in 'normal.

18 Hay $F$, Hull $M$, Torrigian G. The transfer of human IgG subclasses from mother to foetus. Clin Exp Immunol 1971; 9: $355-8$.

19 Oxelious V. IgG subclass levels in infancy and childhood. Acta Paediatrica Scandinavica 1979; 68: 23-7.

20 Rosanelli K. Present diagnostic value of IgM determination in newborns. In: Betke K, Riegel K, Belohradsky BH, eds. Diagnostics in perinatal infections. International Behring Diagnostica Symposium, Hamburg; 1983: 174-8.

21 Conway SP, Dear PRF, Smith I. Immunoglobulin profile of the preterm baby. Arch Dis Child 1985; 60: 208-12.

22 Kaufman HS, Frick OL, Fink D. Serum complement (B1C) in young children with atopic dermatitis. $\mathcal{F}$ Allergy 1968; 42: 1-8.

23 Catty D, Seger R, Drew R, Stroder J, Metze H. IgG-subclass concentrations in cord sera from premature, full class concentrations in cord sera from premature, full 89-96. 